\title{
A NOTE ON CENTRAL MOMENTS IN $C^{*}$-ALGEBRAS
}

\author{
ZOLTÁN LÉKA
}

Abstract. We present sharp estimates of the $k^{\text {th }}$ central moments of normal elements in $C^{*}$ algebras. We shall obtain an upper bound for the weak moments of general elements as well.

Mathematics subject classification (2010): Primary 46L53; Secondary 60B99.

Keywords and phrases: variance, standard deviation, central moments, $C^{*}$-algebra.

\section{REFERENCES}

[1] K. M. R. AUDENAERT, Variance bounds, with an application to norm bounds for commutators, Linear Algebra Appl., 432 (2010), 1126-1143.

[2] B. Aupetit, A Primer on Spectral Theory, Springer-Verlag, 1991.

[3] A. Barvinok, A Course in Convexity, Amer. Math. Soc., 2002.

[4] R. Bhatia AND R. Sharma, Some inequalities for positive linear maps, Linear Algebra Appl., 436 (2012), 1562-1571.

[5] T. Bhattacharyya And P. Grover, Characterization of Birkhoff-James orthogonality, J. Math. Anal. Appl., 407 (2013), 350-358.

[6] J. B. Conway, A Course in Operator Theory, Amer. Math. Soc, Providence, 2000.

[7] M. N. Murthy And V. K. Sethi, Sankhya Ser B, 27 (1965), 201-210.

[8] L. MOLNÁR, Linear maps on observables in von Neumann algebras preserving the maximal deviation, J. Lond. Math. Soc., 81 (2010), 161-174.

[9] G. K. PEDERSEN, Analysis Now, Springer-Verlag, 1989.

[10] M. A. RiefFel, Standard deviation is a strongly Leibniz seminorm, New York J. Math., 20 (2014), $35-56$. 